\title{
Sitzung vom 28. November 1892.
}

\section{Vorsitzender: Vice-Präsident Hr. E. Fischer.}

Das Protocoll der letzten Sitzung wird genehmigt.

Der Vorsitzende begrüsst das in der Sitzang anwesende auswärtige Mitglied, Hrn. Dr. Leppert aus Warschau, sowie Hrn. Prof. Dr. E. Hilgard aus California als Gast.

$\mathrm{Zu}$ ausserordentlichen Mitgliedern werden proclamirt die Herren:

Meyenberg, A., Arcisstr. 1, München;

Schlossmann, Dr. Tb., Brandenburgstr. 1., Berlin S.;

Mehrländer, Dr. H., Berthastr. 18, Hamburg;

Kaufmann, H. Schloss8tr. 57, Stuttgart.

$\mathrm{Zu}$ ausserordentlichen Mitgliedern werden vorgeschlagen die Herren:

Ockel, R., Rheinstr. 61, Friedenau (durch C. Liebermann und A. Bistrzycki);

Gibertini, Prof. Dr. Davis, Parma (durch L. Pesci und G. Ciamician);

Millar, J. H., Mason College, Birmingham (durch J. F. Holtz und R. Daum);

Hilgard, Prof. Dr. E. W., Cniversitüt, California (durch H. Landolt und A. Kossel);

B a u mgärtel, K., Prielmayerstr. 8, München (durch H. Morath und G. Krüs.s);

Steudel, Dr. V., Realanstalt, Reutlingen (durch L. Meyer und $K$. Seubert);

Banse, G., Livienstr. 152, Berlin N (durch S. Gabriel und A. Neumann);

Proromaut, P. M. Linea de Pichi-Ropulli a Osorno, La Uniou, Chile, (durch F. Tiemann und G. Lemme).

Für die Bibliothek sind als Geschenke eingegangen:

699. C. Scheibler. Die Gehaltsernittelung der Zuckerlősungen durch Bestimmung des specifischen Gewichtes derselben bei der Temperatur von $15^{\circ}$. Berlin 1891. 
700. C. Scheib]er. Le titrage des solutions sucrées par la détermination de leurs densités à la température de $15^{0}$. Berlin 1891.

701. Berzelius und Liebig. Ihre Briefe von 1831-1845 mit erlăuternden. Einschaltungen aus gleichzeitigen Briefen von Liebig and Wöhler, sowie wissenschaftlichen Nachweisen. Herausgegeben von Justus. Carriere. München, Leipzig 1893.

Der Vorsitzende:

E. Fischer.
Der Schriftführer:

I. $v$.

W. Will.

\section{Mittheilnngen.}

\section{F. Foerster: Finige Beobachtungen über Kupfer-}

soetatammoniak und Kupferacetatpyridin.

(Vorgetragen vom Verfasser in der Sitzung vom 24. Oktober.)

Im Folgenden soll über einige Beobachtungen Bericht erstattet werden, welche am Kupferacetatammoniak gemacht wurden, da beim Arbeiten mit dieser Verbindung sich die darauf bezüglichen Angaben der Literatur mehrfach als ungenau erwiesen; im Anschluss daran werden einige Versuche beschrieben, welche die Darstellung von Pyridinverbindungen des Kupferacetats zum Gegenstande hatten.

1. Kupferacetatammoniak.

Versetzt man eine wässrige Lösung von Kupferacetat mit überschüssigem Ammoniak und dampft die tiefblaue Lösung anf dem Wasserbade ein, so erhält man schiefwinkelige, schön violett gefärbte Prismen von Kupferacetatammoniak. Bei dieser Art der Darstellung. findet stets eine Abscheidung von sehr fein vertheiltem Kupferoxyd statt, welche sich allerdings leicht auf ein Minimum beschränken lässt. Will man auch dies vermeiden, so löst man unter Zusatz von alkoholischem Ammoniak Kupferacetat in 93-procentigem Alkohol; nach dem Abdestilliren des überschüssigen Alkohols krystallisirt das Kupferacetatammoniak alsbald im Zustande völliger Reinheit aus; das Salz. lässt sich aus starkem Alkohol gut umkrystallisiren, wobei es in kurzen Prismen oder schiefen Octaëdern auftritt, und wird, ohne Zersetzung zu erleiden, in vacuo über Schwefelsäure getrocknet. Seine Zusammensetzung entspricht der Formel

$$
\mathrm{Cu}\left(\mathrm{C}_{2} \mathrm{H}_{3} \mathrm{O}_{2}\right)_{2}, 2 \mathrm{NH}_{3}
$$

